\title{
DURÉE DE PASSAGE DANS L'ÉPIDIDYME DES SPERMATOZOIDES DE VERRAT MARQUÉS AU ${ }^{32} \mathbf{P}$
}

\author{
G SINGH (1) \\ Station de Recherches de Physiologie animale, \\ Centre national de Recherches zootechniques, Jouy-en-Josas (Seine-et-Oise).
}

SOMMAIRE

\begin{abstract}
9 Verrats Large White ont reçu $20 \mathrm{mc}$ de ${ }^{32} \mathrm{PO}_{4} \mathrm{HNa}_{2}$ par voie intraveineuse. Ils ont été castrés le $24^{\mathrm{e}}, 25^{\mathrm{e}}, 26^{\mathrm{e}}, 28^{\mathrm{e}}, 30^{\mathrm{e}}, 32^{\mathrm{e}}$ jour après l'injection. La radioactivité spécifique de l'acide désoxyribonucléique des spermatozoïdes a été déterminée après isolement de ceux-ci dans les différentes parties de l'épididyme.

Les résultats indiquent que les premiers spermatozoïdes à acide désoxyribonucléique radioactif apparaissent dans la partie proximale de la tête de l'épididyme 25 jours après l'injection de ${ }^{32} \mathrm{P}$. Comme on les trouve dans l'éjaculat le $39^{\mathrm{e}}$ jour, on en déduit que la durée du passage dans l'épididyme est de I4 jours. Par ailleurs, on constate que la durée de la spermatogenèse est plus courte, donc plus efficace, chez le Verrat que chez le Bélier et le Taureau.
\end{abstract}

Les spermatozoïdes à acide désoxyribonucléique radioactif apparaissent dans l'éjaculat de Verrat 39 jours après l'injection de ${ }^{32} \mathrm{P}$ (SINGH, I96r a) c'est-à-dire plus tôt que chez le Bélier : $42^{\mathrm{e}}-45^{\mathrm{e}}$ jour (OrTavant, I955; Dawson, I958) et que chez le Taureau : $49^{\mathbf{e}_{-}} \mathbf{2}^{\mathrm{e}}$ jour (DAwson, I958 $b$; KOEFOED-JOHNSEN, I959; ORGEBIN, I96I). Ceci peut résulter soit d'une durée plus courte des processus spermatogénétiques, soit d'un transit épididymaire plus rapide.

La vitesse du transit épididymaire a été étudiée chez plusieurs animaux (Rat, Bélier, Taureau) soit par injection dans le rete testis de particules étrangères (encre de Chine, milieu radioopaque) qui sont supposées progresser à la même vitesse que les spermatozoïdes (GuNN, I936 ; MaC Milian et Harrisson, I955), soit en provoquant la formation d'anomalies spermatiques par isolation scrotale et en déterminant l'apparition de ces spermatozoïdes anormaux dans l'éjaculat (MAC KEnzIE et Philifips, I934), soit enfin en marquant les spermatozoïdes à l'aide de radioisotopes et en suivant la progression de ces spermatozoïdes marqués (ORTAVANT, I954, I959 ;

(r) Adresse actuelle : Division of animal Genetics,

Indian veterinary Research Institute, Izatnagar, L. 1'. (India). 
ORGEBIN, I96I). Cette dernière méthode permet une mesure précise et directe du déplacement des spermatozoïdes. C'est pourquoi nous l'avons choisie pour étudier la durée du transit épididymaire chez le Verrat pour laquelle nous ne possédons aucune donnée, du moins à notre connaissance.

\section{MATÉRIEL E'T MÉTHODE}

Les testicules et épididymes de g Verrats Large White ayant reçu par voie intraveineuse zo mc de ${ }^{32} \mathrm{P}$ sous forme de ${ }^{32} \mathrm{PO}_{4} \mathrm{HNa}_{2}$ ont été prélevés $24,25,26,28,30$, et $3^{2}$ jours après l'injection.

Après enlèvement de l'albuginée et de la majeure partie du tissu conjonctif séparant les différents lobules épididymaires, les épididymes droit et gauche ont été fractionnés en : tête proximale et tête distale, corps, queue proximale et queue distale. Chaque fraction a été coupée en très fins fragments dans $40-50 \mathrm{ml}$ d'une solution froide hypotonique (Krebs-Ringer, $100 \mathrm{ml}+$ eau bidistillée, $400 \mathrm{ml}$ ) et gardée pendant 2 heures à $+5^{\circ} \mathrm{C}$. P'uis elles ont été filtrées sur 6 couches de gaze afin d'éliminer les débris tissulaires. Les filtrats ont été centrifugés et les culots lavés 3 fois avec la même solution hypotonique afin de les débarrasser des hématies. Puis ils ont été honogénéisés et traités selon la technique décrite précédemment ( $\left.\operatorname{SiNGH}_{1} 196 \mathrm{r}\right)$ pour la détermination de la radioactivité spécifique de l'acide désoxyribonucléique.

\section{RÉSULTATS}

Les résultats sont consignés dans les tableaux I et 2 . Ils indiquent que l'activité du résidu spermatique délipidé de la tête proximale de l'épididyme augmente légèrement le $25^{\mathrm{e}}$ jour après $1^{\prime}$ injection, pour atteindre des valeurs élevées dès le $30^{\mathrm{e}}$ jour. Pour le résidu spermatique provenant de la tête distale ceci ne se produit que le $30^{\mathrm{e}}$ jour et pour celui du corps le $32^{\mathrm{e}}$ jour.

Les variations subies par l'activité spécifique de l'acide désoxyribonucléique donnent des indications plus précises. L'activité spécifique de 1'ADN des spermatozoïdes de la partie proximale de la tête de l'épididyme devient significative le $25^{\mathrm{e}}$ jour après l'injection.

TABI,EAU I

Radioactivité du résidu spermatique délipidé des

différentes parties de l'épididyme (coups/min/mg)

\begin{tabular}{|c|c|c|c|c|c|c|c|c|c|c|c|}
\hline \multirow{2}{*}{$\begin{array}{c}\text { Partie } \\
\text { de l'épididyome }\end{array}$} & \multicolumn{11}{|c|}{ Coups/min/mg aux différents jours ajurès l'injection } \\
\hline & & 25 & 25 & 26 & . & 26 & $\quad 28$ & i & 30 & i & 32 \\
\hline 'lête proximale & 8,5 & 2,8 & 12,5 & 11,3 & & $16, \ddot{z}$ & 16,8 & & 41,8 & & 97,8 \\
\hline Tête distale .... & 8,9 & 5,2 & 10,1 & 8,0 & ! & 12,1 & 10,8 & i & 67,0 & $!$ & 105,3 \\
\hline Corps...$\ldots \ldots \ldots$ & 8,9 & 10,3 & 9,8 & 8,0 & & 12,6 & 8,6 & $i$ & 10,8 & 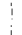 & $9:, 9$ \\
\hline Queue proximale. & 8,5 & 8,7 & $\times, 6$ & 8,0 & & 12,6 & 9,0 & 1 & 9,2 & & 7,0 \\
\hline Queue distale ... & 7,1 & 10,6 & 4,3 & 8,0 & & 12,8 & 8,6 & $i$ & 9,2 & & 6,5 \\
\hline
\end{tabular}




\section{TABLEAU 2}

Radioactivité spécifique de l'acide désoxyribonucléique des spermatosoïdes des différentes parties de l'épididyme à différents moments après l'injection de ${ }^{\mathbf{3 2} P}$

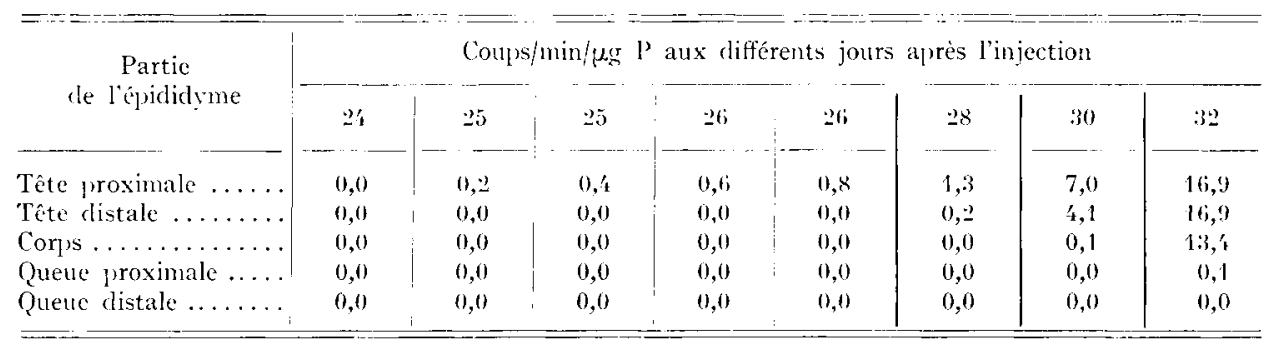

Celle de l'ADN des spermatozoïdes de la partie distale de la tête de l'épididyme le devient le $28^{\mathrm{e}}$ jour, et dans le corps de l'épididyme le $30^{\mathrm{e}}$ jour.

Les premiers spermatozoïdes à ADN radioactif arrivent donc dans l'épididyme le $25^{\circ}$ jour après injection et ces spermatozoïdes progressent ensuite le long du canal épididymaire.

\section{DISCUSSION}

Nous avons montré auparavant (SINGH, I96I) que les spermatozoïdes à ADN radioactif apparaissent dans l'éjaculat 39 jours après l'injection. Or nous constatons ici que ces mêmes spermatozoïdes se trouvent dans la partie proximale de la tête de l'épididyme, 25 jours après l'injection. On peut donc en conclure que ces spermatozoïdes ont mis I4 jours pour traverser l'épididyme.

Ce chiffre est très comparable à celui obtenu chez le Bélier dans des conditions comparables (Or'TAVANT, r955) et il est plus élevé que celui trouvé expérimentalement chez le Taureau, à savoir 8-I I jours (ORGEBin, I96I).

Par contre l'apparition des spermatozoïdes radioactifs dans la tête de l'épididyme le $25^{\mathrm{e}}$ jour après l'injection est plus précoce que chez le Bélier, $30^{\mathrm{e}}$ jour, et beaucoup plus que chez le Taureau, $4 \mathrm{I}^{\mathrm{e}}$ jour. La durée de la spermatogenèse est donc beaucoup plus courte chez le Verrat que chez les deux autres animaux domestiques. C'est cette particularité qui explique que les spermatozoïdes à $A D N$ radioactifs apparaissent plus tôt dans l'éjaculat chez ces Verrats que chez les autres animaux domestiques étudiés.

\section{REMERCIEMENTS}

L'auteur remercie R. ORTAVANT, Directeur de Recherches à l'Institut national de la Recherche agronomique pour lui avoir suggéré ce travail et l'avoir guidé tout au long de sa réalisation.

('e travail a pu être effectué grâce à deux bourses du gouvernement français. 


\section{SUMMARY}

DURATION OF I'ASSING THROUGH THE EPIDIDYMIS OF ${ }^{32} \mathrm{P}^{2}$ LABELIEI BOAR SPLRMATOZOA

I. Six young Boars were injected each with $20 \mathrm{mc}$ of radioactive phosphate ( $\left.{ }^{32} \mathrm{P}\right)$ intravenously and were castrated on different days, 24th, $25^{\text {th }}, 26$ th, 3 oth and 32 days after the injection.

2. The epididymides were separated and cleared off excess connective tissue. Fach epididymis was cut into five portions, the proximal and the distal parts of the head and the tail and the body of the epididymis. Wach portion was then cut into small pieces and soaked in hypotonic solution at $5^{\circ} \mathrm{C}$ in a refrigerator for two hours to allow maceration then filtered.

3. The filtrate containing sperm was centrifuged and the residue homogenised and treated by the standard technique employed for the extraction of desoxyribonucleic acid.

4. The specific activities of the residue sperm and DNA fraction of sperm recovered from different parts of the epididymis were estimated.

5. The specific activity in the DNA fraction of sperm appeared on the 25th day after the injection.

From this finding and the results of the earlier investigation showing the appearance of activity at first in the sperm desoxyribonucleic acid of the ejaculate ( 39 th days), it is estimated that the sperm will take 44 days to travel through the epididvmal canal in Boar.

\section{RÉFÉRENCES BIBLIOGRAPHIQUES}

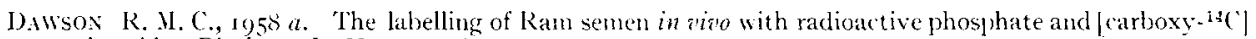
stearic acid. Biochem. J., 68, 512-518.

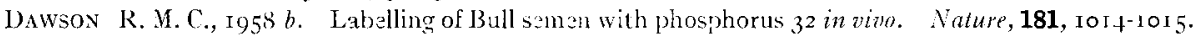

GUNN R. M. C., I936. Fertility in sheep, artificial production of seminal ejaculation and the rharacters spermatozoa contained there in. Bull. Counc. Sci. Industr. Res. Aust., 94, 5 I.

KOEFOEI-JOHNSEN H. H., I95\%. Influence of ejaculation frequency on the time required for sperm formation and epididymal passage in the Bull. Nalure, 185, 49-50.

Il Kenzie F. F., Pinllifipi R. W., I934. Thermo-regulatory function and mechanism of the scrotum. Res. Bull. Mo. agric. Exp. Sta., 217, I-73.

Mac Millay E. W., Harrison R. Gi, I955. The rate of passage of radioopaque medium along the ductus epididymides of the Rat. Proc. Soc. Stud. Fert., 7, 35-40.

OrGeBIN M. C., rg6r. Étude du transit épididymaire des spermatozö̈des de Taureau marqués à l'aide du ${ }^{32} \mathrm{P}$. Ann. Biol. anim. Bioch. Biophys., 1, i 1 7 - 20.

Ortavant R., r954. Dítermination de la vitessa du trunsfert des spermatozoïd as dans l'épididyme de Bélier it l'aide de ${ }^{32}$ P. C. R. Soc. Biol., 148, 866-868.

OrTavant R., I955. Etude sur la spermatogenèse des animaux domestiques à l'aide du phosphore-32. Cont. int. utilis. energie atomique ḋ des fins pacifizuos, $\mathbf{1 2}, 2+3-2+5$.

Ortavant R., I95\%. La cycle s'srmatogénétique chez le Bélier. Inn. Zootech., 8, I83-244, 27 1-321.

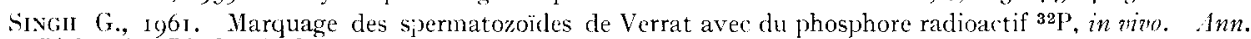
Biol. anim. Bioch. Biophys., 1, 40.3-405. 